\title{
EFFECT OF SORET AND TEMPERATURE DEPENDENT VISCOSITY ON THERMOHALINE CONVECTION IN A FERROFLUID SATURATING A POROUS MEDIUM
}

\author{
R. SEKAR ${ }^{*}$ and K. RAJU \\ Department of Mathematics \\ Pondicherry Engineering College \\ Puducherry - 605 014, INDIA \\ E-mail: rsekar@pec.edu
}

\begin{abstract}
Soret driven ferrothermoconvective instability in multi-component fluids has a wide range of applications in heat and mass transfer. This paper deals with the theoretical investigation of the effect of temperature dependent viscosity on a Soret driven ferrothermohaline convection heated from below and salted from above subjected to a transverse uniform magnetic field in the presence of a porous medium. The Brinkman model is used in the study. It is found that the stationary mode of instability is preferred. For a horizontal fluid layer contained between two free boundaries an exact solution is examined using the normal mode technique for a linear stability analysis. The effect of salinity has been included in magnetization and density of the fluid. The critical thermal magnetic Rayleigh number for the onset of instability is obtained numerically for sufficiently large values of the buoyancy magnetization parameter $M_{1}$ using the method of numerical Galerkin technique. It is found that magnetization and permeability of the porous medium destabilize the system. The effect of temperature dependent viscosity stabilizes the system on the onset of convection.
\end{abstract}

Keywords: ferroconvection, porous medium, Soret effect, multi-component system, Brinkman model, temperature dependent viscosity, Galerkin technique.

\section{Introduction}

Magnetic fluids, also called 'ferrofluids', are electrically non-conducting colloidal suspensions of tiny particles of solid ferromagnetic material in a non-electrically conducting carrier fluid like water or heptanes, kerosene, hydrocarbon, etc. These fluids behave as a homogeneous continuum and exhibit a variety of interesting phenomena. Ferromagnetic fluids are not found in nature but are artificially synthesized. The viscosity of a magnetic nanofluid as a function of the applied magnetic field, direction of magnetic field with respect to the flow direction and temperature, is useful for endurable applications for magnetic inkjet printers, heat transfer, nanomotors, nanogeneratores, inertial dampers, switches, sensors, transformer cooling, loudspeaker, similar micro-and nanofluidic devices, magnetic targeted drug delivery, cancer treatment in biomedicine field, etc. (Odenbach and Thurm, 2012), (Berkovsky and Bastovoy, 1996) and (Gazeau et al., 1997).

An introduction to the research on magnetic fluids has been given in the monograph by Rosensweig (1985), which reviews several applications of heat transfer through ferrofluids, such as enhanced convective cooling having a temperature dependent magnetic moment due to magnetization of the fluid. This magnetization is called ferroconvection, which is similar to Bénard convection (Chandrasekhar, 1985). Convective instability of ferromagnetic fluids has been predicted by Finlayson (1970). Schwab et al. (1983) investigated experimentally the Finlayson's problem in the case of a strong magnetic field and detected the onset of convection by plotting the Nusselt number versus the Rayleigh number. Then, the critical Rayleigh

\footnotetext{
* To whom correspondence should be addressed
} 
number corresponds to a discontinuity in the slope. Later, Stiles and Kagan (1990) examined the experimental problem reported by Schwab et al. (1983) and generalized Finlayson's model assuming that under a strong magnetic field, the rotational viscosity augments the shear viscosity. Furthermore, Vaidyanathan et al., (1991) investigated the theoretically the convective instability of a ferromagnetic fluid in a porous medium of large permeability by use of the Brinkman model. This investigation has been done to the effect of temperature dependent viscosity by Ramanathan and Muchikel (2006) using Galerkin technique.

Suresh Govindan et al. (2012) made a numerical analysis on ferroconvection with temperature dependent viscosity and anisotropic porous medium. Nanjundappa et al. (2012) introduced the effect of temperature dependent viscosity on Marangoni-Bénard ferroconvection without a porous medium under microgravity conditions in a horizontal ferrofluid layer in the presence of a uniform vertical magnetic field. Moreover, this work has been analyzed to the effect magnetic field dependent viscosity in the absence of temperature dependent viscosity by Nanjundappa et al. (2010). They used the Rayleigh Ritz method with Chebyshev polynomials of second kind as trial function. The onset of buoyancy-driven convection in a ferromagnetic fluid in the presence of a porous medium was studied by Shivakumara et al. (2010). The thermorheological effect of magnetoconvection in fluids with weak electrical conductivity was studied numerically by Siddheshwar (2004).

The study of convection in two component ferrofluids will throw light on convective instability. This is referred to as a type of convection known as ferrothermohaline convection studied by Baines and Gill (1969). Vaidyanathan et al. $(1995$; 1997) investigated the presence and absence of a porous medium on ferrothermohaline. Here, they found that the salinity of a ferromagnetic fluid enabled the fluid to destabilize more when it is salted from above. A really interesting situation from both a geophysical and a mathematical viewpoint arises when the layer is simultaneously heated from below and salted from above. Sunil et al. (2011) considered the double-diffusive convection on ferromagnetic fluid with rotation and internal angular momentum.

Recently, Vaidyanathan et al. (2005) attempted to study the Soret effect due to ferrothermohaline convection of a sparse distribution. Further, the condition of a porous medium of ferroconvective instability of multi-component fluid heated from below and salted from above was analyzed by Sekar et al. (2006; 2013) for isotropic and anisotropic models. The effect of rotation on thermohaline convection in a ferromagnetic fluid saturating an anisotropic porous medium with the Soret effect was obtained by Sekar et al. (2013a) and this investigation was carried out for magnetic field dependent viscosity by Sekar and Raju (2013).

In view of these investigations, it is attempted to analyze the effect of temperature dependent viscosity on the Soret driven ferrothermohaline convection in the presence of an isotropic porous medium of low permeability, subjected to a vertical magnetic field using the Brinkman model. In this investigation, the free boundaries are considered. The resulting eigen value problem is solved numerically using the Galerkin method. Besides, an analytical formula is obtained for the critical magnetic Rayleigh number by a regular perturbation method.

\section{Mathematical formulation}

In this investigation, we consider an infinite spread horizontal layer of an Oberbeck-Boussinesq ferromagnetic fluid of thickness " $d$ " saturating a sparsely distributed porous medium heated from below and salted from above. The temperature and salinity at the bottom and top surfaces are $z= \pm d / 2$ are $T_{0} \pm \Delta T / 2$ and $S_{0} \pm \Delta S / 2$, respectively. Both the boundaries are assumed to be free and perfect conductors of heat and salt. This fluid layer is taken to be an isotropic porous medium and the fluid viscosity is assumed to be temperature-dependent in the following form (Ramanathan and Muchikel, 2006 and Siddheshwar, 2004)

$$
\mu(T)=\mu_{1}\left[1-\delta\left(T-T_{a}\right)^{2}\right]
$$


where $\delta$ is a small positive quantity.

The gravity field $\boldsymbol{g}=(0,0,-g)$ and uniform vertical magnetic field intensity $\boldsymbol{H}=\left(0,0, H_{0}\right)$ pervade the system. Considering the Soret effect on the temperature gradient the mathematical equations governing the above investigation are as follows.

The continuity equation for an incompressible fluid is

$$
\nabla \cdot \boldsymbol{q}=0
$$

The corresponding momentum equation is

$$
\rho_{0} \frac{D \boldsymbol{q}}{D t}=-\nabla p+\rho \boldsymbol{g}+\nabla \cdot(\boldsymbol{H B})+\nabla\left[\mu(T)\left(\nabla \boldsymbol{q}+\nabla \boldsymbol{q}^{T r}\right)\right]-\frac{\mu(\mathrm{T})}{k} \boldsymbol{q} .
$$

The temperature equation for an incompressible ferromagnetic fluid is

$$
\left[\rho_{0} C_{v, H}-\mu_{o} \boldsymbol{H}(\partial \boldsymbol{M} / \partial T)_{v, H}\right](d T / d t)+\mu_{0} T(\partial \boldsymbol{M} / \partial T)_{v, H} \cdot(d \boldsymbol{H} / d t)=K_{l} \nabla^{2} T+\phi
$$

The conservation of mass flux equation is given by

$$
\rho_{0}(\partial / \partial t+\boldsymbol{q}) S=K_{S} \nabla^{2} S+S_{T} \nabla^{2} T
$$

The density equation of state for a Boussinesq two-component fluid is

$$
\rho=\rho_{0}\left[1-\alpha_{t}\left(T-T_{0}\right)+\alpha_{S}\left(S-S_{0}\right)\right] .
$$

Maxwell's equations, simplified for a non-conducting fluid with no displacement currents, become

$$
\nabla \cdot \boldsymbol{B}=0, \quad \nabla \times \boldsymbol{H}=0
$$

where the magnetic induction is given by

$$
\boldsymbol{B}=\mu_{0}(\boldsymbol{M}+\boldsymbol{H}) .
$$

In general, the pressure of ferromagnetic fluid can distort an external magnetic field if a magnetic interaction (dipole-dipole) takes place, but this is negligible for small particle concentration, as is assumed here. We assume that the magnetization is aligned with the magnetic field, but allows a dependence on the magnitude of the magnetic field, temperature and salinity, so that

$$
\boldsymbol{M}=\frac{\boldsymbol{H}}{H} M(H, T, S) .
$$

The magnetic equation of state is linearized about the magnetic field $H_{0}$, an average temperature $T_{0}$ and an average salinity $S_{0}$, to become

$$
M=M_{0}+\chi\left(\mathrm{H}-H_{0}\right)-K\left(T-T_{0}\right)+K_{2}\left(S-S_{0}\right) .
$$


Here $H_{0}$ is the uniform magnetic field of the fluid layer when placed in an external magnetic field $\boldsymbol{H}=H_{0}^{\text {ext }} \boldsymbol{k}, \boldsymbol{k}$ is a unit vector in the $z$-direction, $H=|\boldsymbol{H}|, M=|\boldsymbol{M}|$ and $M_{0}=\left(H_{0}, T_{0}, S_{0}\right)$.

The basic state is assumed to be quiescent and is given by

$$
\begin{aligned}
& \boldsymbol{q}=\boldsymbol{q}_{b}=(0,0,0), \quad T=T_{b}=T_{0}-\beta_{t} z, \quad S=S_{b}=S_{0}-\beta_{S} z, \quad \rho(z)=\rho_{0}\left[1+\alpha_{t} \beta_{t} z-\alpha_{S} \beta_{S} z\right] \\
& p=p_{b}(z), \quad H_{b}(z)=\left[H_{0}-\frac{K \beta_{t} z}{1+\chi}+\frac{K_{2} \beta_{S} z}{1+\chi}\right] \boldsymbol{k}, \quad M_{b}(z)=\left[M_{0}+\frac{K \beta_{t} z}{1+\chi}-\frac{K_{2} \beta_{S} z}{1+\chi}\right] \boldsymbol{k}
\end{aligned}
$$

where $\boldsymbol{k}$ is the unit vector in the vertical direction, $\beta_{t}$ and $\beta_{S}$ are non-negative constants.

Moreover, the basic state is disturbed by an infinitesimal thermal perturbation. Let the component of the perturbed magnetization and the magnetic field be $\left(M_{1}^{\prime}, M_{2}^{\prime}, M_{b}(z)+M_{3}^{\prime}\right)$ and $\left(H_{1}^{\prime}, H_{2}^{\prime}, H_{b}(z)+H_{3}^{\prime}\right)$, respectively. The perturbed viscosity and temperature are taken as $\mu_{b}(z)+\mu^{\prime}$ and $T_{b}(z)+T^{\prime}$, respectively. On linearization, and assuming $K \beta_{t} d<<(1+\chi) H_{0}$ and $K_{2} \beta_{S} d<<(1+\chi) H_{0}$ and using the expressions for $H_{b}$ and $M_{b}$ in Eqs (2.11), Eqs (2.7)-(2.9) become

$$
\begin{aligned}
& H_{i}^{\prime}+M_{i}^{\prime}=\left[1+\left(M_{0} / H_{0}\right)\right] H_{i}^{\prime}, \quad \text { for } \quad i=1,2, \\
& H_{3}^{\prime}+M_{3}^{\prime}=(1+\chi) H_{3}^{\prime}-K_{2} S+S_{T} K \theta-K \theta, \\
& B_{i}^{\prime}=\mu_{0}\left[1+\left(M_{0} / H_{0}\right)\right] H_{i}^{\prime}, \quad \text { for } \quad i=1,2, \\
& B_{3}=\mu_{0}\left(M_{0}+H_{0}\right)+\mu_{0}(1+\chi) H_{3}^{\prime}-\mu_{0} K_{2} S+\mu_{0} S_{T} K \theta-\mu_{0} K \theta .
\end{aligned}
$$

Equation (2.7b) implies that $\boldsymbol{H}^{\prime}=\nabla \phi^{\prime}$, where $\phi^{\prime}$ is the perturbed magnetic potential and using the analyses of Sekar et al., (2013; 2013a), the vertical component of the momentum equation can be written as

$$
\begin{aligned}
& \rho_{0} \frac{\partial}{\partial t}\left(\nabla^{2} w\right)=\rho_{0} g \alpha_{t} \nabla_{l}^{2} T^{\prime}-\rho_{0} g \alpha_{S} \nabla_{l}^{2} S^{\prime}-K \beta_{t} \frac{\partial}{\partial z}\left(\nabla_{l}^{2} \phi^{\prime}\right)+\frac{\mu_{0} K^{2} \beta_{t}\left(1-S_{T}\right)}{1+\chi} \nabla_{l}^{2} T^{\prime}+ \\
& -\frac{\mu_{0} K K_{2} \beta_{S}\left(1-S_{T}\right)}{1+\chi} \nabla_{l}^{2} T^{\prime}-\frac{\mu_{0} K K_{2} \beta_{t}}{1+\chi} \nabla_{I}^{2} S^{\prime}+\frac{\mu_{0} K^{2} \beta_{S}}{1+\chi} \nabla_{l}^{2} S^{\prime}+\mu_{0}\left(\nabla^{2}\left(\nabla^{2} w\right)\right)+ \\
& -\mu_{0} K_{2} \beta_{S} \frac{\partial}{\partial z}\left(\nabla_{l}^{2} \phi^{\prime}\right)-\frac{\mu_{b}}{k} \nabla^{2} w+\frac{\partial^{2} \mu_{b}}{\partial z^{2}} \nabla^{2} w+2 \frac{\partial \mu_{b}}{\partial z} \frac{\partial}{\partial z}\left(\nabla^{2} w\right)-\frac{1}{k} \frac{\partial \mu_{b}}{\partial z}\left(\frac{\partial w}{\partial z}\right) .
\end{aligned}
$$

The modified Fourier heat conduction equation is

$$
\rho_{0} C_{v, H} \frac{\partial \theta}{\partial t}-\mu_{0} K T_{0} \frac{\partial}{\partial t}\left(\frac{\partial \phi}{\partial z}\right)=K_{l}\left(\nabla^{2} \theta\right)+\left[\rho_{0} c \beta_{t}-\left(\frac{\mu_{0} K^{2} T_{0}^{2} \beta_{t}}{1+\chi}\right)+\left(\frac{\mu_{0} K K_{2} T_{0} \beta_{S}}{1+\chi}\right)\right] w
$$

where

$$
\rho_{0} C=\rho_{0} C_{v, H}+\rho_{0} K H_{0} .
$$

The salinity equation is 


$$
\frac{\partial S}{\partial t}+\beta_{S} w=K_{S}\left(\nabla^{2} S\right)+S_{T}\left(\nabla^{2} \theta\right)
$$

Using the analysis similar to Sekar et al. (2013) one gets

$$
(1+\chi) \frac{\partial^{2} \phi}{\partial z^{2}}+\left(1+\frac{M_{0}}{H_{0}}\right) \nabla_{l}^{2} \phi-K \frac{\partial \theta}{\partial z}+K_{2} \frac{\partial S}{\partial z}+S_{T} K \frac{\partial \theta}{\partial z}=0
$$

where

$$
\nabla_{l}^{2}=\left(\partial^{2} / \partial x^{2}\right)+\left(\partial^{2} / \partial y^{2}\right) \quad \text { and } \quad \nabla^{2}=\nabla_{l}^{2}+\left(\partial^{2} / \partial z^{2}\right)
$$

\section{Normal mode analysis method}

Analyzing the small thermal disturbances into normal modes, we assume that the perturbation quantities are of the form

$$
(w, T, \phi, S)=\left[w(z, t), T(z, t), \phi^{\prime}(z, t), S^{\prime}(z, t)\right] \exp \left(i\left(k_{x} x+k_{y} y\right)\right)
$$

where $k_{0}=\sqrt{k_{x}^{2}+k_{y}^{2}}$ is the resultant wave number, $k_{x}$ is the wave number along the $x$ direction and $k_{y}$ is the wave number along the $y$ direction.

Following the normal mode analysis, the linearized perturbation dimensionless equations

$$
\begin{aligned}
& \frac{\partial}{\partial t^{*}}\left(D^{2}-a^{2}\right) w^{*}=a R^{1 / 2}\left[M_{1} D \phi^{*}-\left(1+M_{1}\left(1-S_{T}\right) T^{*}\right)\right]+M_{1} M_{5} a R^{1 / 2} D \phi^{*}+ \\
& -M_{1} M_{5} a R^{1 / 2}\left(1-S_{T}\right) T^{*}+\left(D^{2}-a^{2}\right)^{2} w^{*}+a R_{S}^{1 / 2}\left[1+M_{4}+M_{4} M_{5}^{-1}\right] S^{*}+ \\
& +\left(1-V z^{2}\right)\left(D^{2}-a^{2}\right)^{2} w^{*}-\frac{\left(1-V z^{*}\right)}{k^{*}}\left(D^{2}-a^{2}\right) w^{*}-2 V\left(D^{2}-a^{2}\right) w^{*}+ \\
& -4 V z *\left(D^{2}-a^{2}\right) D w^{*}+\frac{2 V z^{*}}{k^{*}} D w^{*}, \\
& \mathrm{P}_{\mathrm{r}}\left[\frac{\partial T^{*}}{\partial t^{*}}-M_{2} \frac{\partial}{\partial t^{*}}\left(D \phi^{*}\right)\right]=\left(D^{2}-a^{2}\right) T^{*}+a R^{1 / 2}\left(1-M_{2}-M_{2} M_{5}\right) w^{*}, \\
& \mathrm{P}_{\mathrm{r}} \frac{\partial S^{*}}{\partial t^{*}}=\tau\left(D^{2}-a^{2}\right) S^{*}-a R_{S}^{1 / 2} M_{6} w^{*}+S_{T} M_{5} M_{6}^{-1}\left(R / R_{S}\right)^{1 / 2}\left(D^{2}-a^{2}\right) T^{*}, \\
& D^{2} \phi^{*}-M_{3} a^{2} \phi^{*}-\left(1-S_{T}\right) D T^{*}+M_{5} M_{6}^{-1}\left(R / R_{S}\right)^{1 / 2} D S^{*}=0
\end{aligned}
$$

where the following non-dimensional parameters are introduced 


$$
\begin{aligned}
& w^{*}=\frac{w d}{v}, \quad t^{*}=\frac{v t}{d^{2}}, \quad T^{*}=\left(\frac{K_{1} a R^{1 / 2}}{\rho_{0} C_{v, H} \beta_{t} v d}\right) \theta, \quad \phi^{*}=\left(\frac{(1+\chi) K_{l} a R^{1 / 2}}{\rho_{0} C_{v, H} K \beta_{t} v d^{2}}\right) \phi, \\
& z^{*}=\frac{z}{d}, \quad a=k_{0} d, \quad D=\frac{\partial}{\partial z^{*}}, \quad S^{*}=\left(\frac{K_{S} a R_{S}^{1 / 2}}{\rho_{0} C_{v, H} \beta_{S} v d}\right) S, \quad v=\frac{\mu}{\rho_{0}}, \quad k^{*}=\frac{k}{d^{2}}, \\
& M_{1}=\frac{\mu_{0} K^{2} \beta_{t}}{(1+\chi) \rho_{0} g \alpha_{t}}, \quad M_{2}=\frac{\mu_{0} K^{2} T}{(1+\chi) \rho_{0} C_{v, H}}, \quad M_{3}=\frac{1+\left(M_{0} / H_{0}\right)}{(1+\chi)}, \\
& M_{4}=\frac{\mu_{0} K^{2} \beta_{S}}{(1+\chi) \rho_{0} g \alpha_{S}}, \quad M_{5}=\frac{K_{2} \beta_{S}}{K \beta_{t}}, \quad M_{6}=\frac{K_{S}}{K_{l}}, \quad \mathrm{P}_{\mathrm{r}}=\frac{\mu C_{v, H}}{K_{1}}, \\
& R=\frac{\rho_{0} C_{v, H} \beta_{t} \alpha_{t} g d^{4}}{v K_{1}}, \quad R_{S}=\frac{\rho_{0} C_{v, H} \beta_{S} \alpha_{S} g d^{4}}{v K_{S}}, \quad \tau=\rho_{0} C_{v, H}\left(\frac{K_{S}}{K_{l}}\right)
\end{aligned}
$$

where $R$ is the thermal Rayleigh number, $R_{S}$ is the salinity Rayleigh number, Pr is the Prandtl number.

\section{Exact solution for free boundaries using Galerkin technique}

Here the simplest boundary conditions chosen, namely free-free, isothermal with infinite magnetic susceptibility $\chi$ in the perturbed field keep the problem analytically tractable and serve the purpose of providing a qualitative insight into the problem. The case of two free boundaries is of little physical interest, but it is mathematically important because one can derive an exact solution, whose properties guide our analysis. Thus the exact solution of the system subjected to the boundary conditions

$$
w^{*}=D^{2} w^{*}=T^{*}=D \phi^{*}=S^{*}=0 \quad \text { at } \quad z^{*}=-1 / 2 \quad \text { and } \quad z^{*}=+1 / 2 .
$$

is written in the form

$$
\begin{aligned}
& w^{*}=A w_{l}(z) e^{\sigma t^{*}} \cos \pi z^{*}, \quad T^{*}=B T_{1}(z) e^{\sigma t^{*}} \cos \pi z^{*}, \quad S^{*}=C S_{l}(z) e^{\sigma t^{*}} \cos \pi z^{*}, \\
& D \phi^{*}=F \phi_{1}(z) e^{\sigma t^{*}} \cos \pi z^{*}, \quad \phi^{*}=\frac{F}{\pi} \phi_{l}(z) e^{\sigma t^{*}} \sin \pi z^{*}
\end{aligned}
$$

Substituting Eqs (4.2) in linearized perturbation dimensionless Eqs (3.1)-(3.4) and dropping asterisks for convenience, we get the following equations

$$
\begin{aligned}
& \left\{\left(\sigma+\left(\left(1-V z^{2}\right) / k\right)\right)\left(D^{2}-a^{2}\right)^{2} w_{l}(z)-\left(1-V z^{2}\right)\left(D^{2}-a^{2}\right)^{2} w_{l}(z)+\right. \\
& \left.+2 V\left(D^{2}-a^{2}\right)\left[1+2 z D w_{l}(z)\right]-\frac{1}{k} 2 v z D w_{l}(z)\right\} A+a R^{1 / 2}\left[1+M_{l}\left(1+M_{5}\right)\left(1-S_{T}\right) T_{1}(z)\right] B+(4.3) \\
& -a R_{S}^{1 / 2}\left(1+M_{4}+M_{4} M_{5}^{-1}\right) S_{l}(z) C+a R^{1 / 2} M_{1}\left(1+M_{5}\right) D \phi_{l}(z) F=0
\end{aligned}
$$




$$
\begin{aligned}
& a R^{1 / 2}\left(1-M_{2}-M_{2} M_{5}\right) w_{l}(z) A+\left(D^{2}-a^{2}-\mathrm{P}_{\mathrm{r}} \sigma\right) T_{l}(z) B+\mathrm{P}_{\mathrm{r}} \sigma M_{2} D \phi_{l}(z) F=0, \\
& -a R_{S}^{1 / 2} M_{6} w_{l}(z) A+S_{T} M_{5} M_{6}^{-1}\left(R / R_{S}\right)^{1 / 2}\left(D^{2}-a^{2}\right) T_{l}(z) B+ \\
& +\left[\tau\left(D^{2}-a^{2}\right)-\sigma \mathrm{P}_{\mathrm{r}}\right] S_{l}(z) C=0, \\
& -R_{S}^{1 / 2} \pi^{2}\left(1-S_{T}\right) D T_{l}(z) B+R^{1 / 2} M_{5} M_{6}^{-1} D S_{l}(z) C+R_{S}^{l / 2}\left(D^{2}-a^{2} M_{3}\right) \phi_{l}(z) F=0,
\end{aligned}
$$

For the existence of non-trivial solutions, the determinant of the coefficients of $A, B, C$ and $F$ must vanish. This determinant on simplification yields

$$
-T_{1} \sigma^{3}+T_{2} \sigma^{2}+T_{3} \sigma+T_{4}=0
$$

where

$$
\begin{aligned}
& T_{1}=\left\langle\mathrm{P}_{\mathrm{r}} S_{1}\left\langle\mathrm{P}_{\mathrm{r}} T_{1} b_{1} w_{1}\right\rangle b_{5} \phi_{1}\right\rangle \\
& T_{2}=\left\langle\mathrm{P}_{\mathrm{r}} T_{1}\left\langle\tau b_{1} S_{1} b_{1} w_{1}\right\rangle b_{5} \phi_{1}\right\rangle-\left\langle b_{1} T_{1}\left\langle\mathrm{P}_{\mathrm{r}} S_{1} b_{1} w_{1}\right\rangle b_{5} \phi_{1}\right\rangle \\
& T_{3}=-\left\langle b_{1} T_{1}\left\langle\tau b_{1} S_{1} b_{1} w_{1}\right\rangle b_{5} \phi_{1}\right\rangle+\left\langle\left(1-V z^{2}\right) \frac{b_{1}}{k} w_{1}\left\langle\mathrm{P}_{\mathrm{r}} T_{1} \tau b_{1} S_{1}\right\rangle b_{5} \phi_{1}\right\rangle+ \\
& -\left\langle\left(1-V z^{2}\right) b_{1}^{2} w_{1}\left\langle\mathrm{P}_{\mathrm{r}} T_{1} \tau b_{1} S_{1}\right\rangle b_{5} \phi_{1}\right\rangle+\left\langle 2 V b_{1}\left(1+2 z D w_{1}\right)\left\langle\mathrm{P}_{\mathrm{r}} T_{1} \tau b_{1} S_{1}\right\rangle b_{5} \phi_{1}\right\rangle+ \\
& -\left\langle\frac{2 V z}{k} D w_{1}\left\langle\mathrm{P}_{\mathrm{r}} T_{1} \tau b_{1} S_{1}\right\rangle b_{5} \phi_{1}\right\rangle+a^{2} R\left\langle b_{2} D \phi_{1}\left\langle\mathrm{P}_{\mathrm{r}} T_{1} M_{6} w_{1}\right\rangle b_{4} D S_{1}\right\rangle+ \\
& -\left\langle\left(1-V z^{2}\right) \frac{b_{1}}{k} w_{1}\left\langle b_{1} T_{1} \mathrm{P}_{\mathrm{r}} S_{1}\right\rangle b_{5} \phi_{1}\right\rangle+a^{2} R_{S}\left\langle b_{3} S_{1}\left\langle\mathrm{P}_{\mathrm{r}} T_{1} M_{6} w_{1}\right\rangle b_{5} \phi_{1}\right\rangle+ \\
& +\left\langle\left(1-V z^{2}\right) b_{1}^{2} w_{1}\left\langle b_{1} T_{1} \mathrm{P}_{\mathrm{r}} S_{1}\right\rangle b_{5} \phi_{1}\right\rangle-\left\langle 2 V b_{1}\left(1+2 z D w_{1}\right)\left\langle b_{1} T_{1} \mathrm{P}_{\mathrm{r}} S_{1}\right\rangle b_{5} \phi_{1}\right\rangle+ \\
& +\left\langle\frac{2 V z}{k} D w_{1}\left\langle b_{1} T_{1} \mathrm{P}_{\mathrm{r}} S_{1}\right\rangle b_{5} \phi_{1}\right\rangle+a^{2} R\left\langle\left(1+b_{2}\left(1-S_{T}\right) T_{1}\right)\left\langle w_{1} \mathrm{P}_{\mathrm{r}} S_{1}\right\rangle b_{5} \phi_{1}\right\rangle+ \\
& +a^{2} R\left\langle b_{2} D \phi_{1}\left\langle w_{1}\left(1-S_{T}\right) D T_{1}\right\rangle \mathrm{P}_{\mathrm{r}} S_{1}\right\rangle, \\
& T_{4}=-\left\langle\left(1-V z^{2}\right) \frac{b_{1}}{k} w_{1}\left\langle b_{1} T_{1} \tau b_{1} S_{1}\right\rangle b_{5} \phi_{1}\right\rangle+\left\langle\left(1-V z^{2}\right) b_{1}^{2} w_{1}\left\langle b_{1} T_{1} \tau b_{1} S_{1}\right\rangle b_{5} \phi_{1}\right\rangle+ \\
& -\left\langle 2 V b_{1}\left(1+2 z D w_{1}\right)\left\langle b_{1} T_{1} \tau b_{1} S_{1}\right\rangle b_{5} \phi_{1}\right\rangle+\left\langle\frac{2 V z}{k} D w_{1}\left\langle b_{1} T \quad b_{5} \phi_{1}\right\rangle \tau b_{1} S_{1}\right\rangle+ \\
& +a^{2} R\left\langle\left(1+b_{2}\left(1-S_{T}\right) T_{1}\right)\left\langle w_{1} \tau b_{1} S_{1}\right\rangle b_{5} \phi_{1}\right\rangle+a^{2} R_{S}\left\langle b_{3} S_{1}\left\langle w_{1} S_{T} b_{1} b_{4} T_{1}\right\rangle b_{5} \phi_{1}\right\rangle+ \\
& +a^{2} R_{S}\left\langle b_{3} S_{1}\left\langle\mathrm{P}_{\mathrm{r}} T_{1} M_{6} w_{1}\right\rangle b_{5} \phi_{1}\right\rangle+a^{2} R\left\langle b_{2} D \phi_{1}\left\langle w_{1} S_{T} b_{1} b_{4} T_{1}\right\rangle b_{4} D S_{1}\right\rangle+ \\
& +a^{2} R\left\langle b_{2} D \phi_{1}\left\langle w_{1}\left(1-S_{T}\right) D T_{1}\right\rangle \tau b_{1} S_{1}\right\rangle+a^{2} R\left\langle b_{2} D \phi_{1}\left\langle b_{1} T_{1} M_{6} w_{1}\right\rangle b_{4} D S_{1}\right\rangle,
\end{aligned}
$$




$$
\begin{aligned}
& b_{1}=D^{2}-a^{2}, \quad b_{2}=M_{1}\left(1+M_{5}\right), \quad b_{3}=1+M_{4}+\left(M_{4} / M_{5}\right), \\
& b_{4}=\left(M_{5} / M_{6}\right) \quad \text { and } \quad b_{5}=D^{2}-a^{2} M_{3} .
\end{aligned}
$$

For obtaining stationary instability, the time-dependent term $T_{4}$ is equal to zero. From Eq.(4.7) it is easy to obtain the eigen value $R_{c}$.

$$
R_{c}=\frac{x_{1}-a^{2} R_{S}\left(x_{2} S_{T}+x_{3}\right)}{\tau x_{4}+M_{1}\left(1+M_{5}\right)\left[\left(1-S_{T}\right) \tau x_{5}+x_{6} S_{T}+\tau\left(1-S_{T}\right) x_{7}+x_{8}\right]}
$$

where

$$
\begin{aligned}
& x_{1}=-\left\langle\left(1-V z^{2}\right) \frac{b_{1}}{k} w_{1}\left\langle b_{1} T_{1} \tau b_{1} S_{1}\right\rangle b_{5} \phi_{1}\right\rangle+\left\langle\left(\begin{array}{ll}
1-V z^{2}
\end{array}\right) b_{1}^{2} w_{1}\left\langle b_{1} T_{1} \tau b_{1} S_{1}\right\rangle b_{5} \phi_{1}\right\rangle+ \\
& -\left\langle 2 V b_{1}\left(1+2 z D w_{1}\right)\left\langle b_{1} T_{1} \tau b_{1} S_{1}\right\rangle b_{5} \phi_{1}\right\rangle+\left\langle\frac{2 V z}{k} D w_{1}\left\langle b_{1} T \quad b_{5} \phi_{1}\right\rangle \tau b_{1} S_{1}\right\rangle \\
& x_{2}=\left\langle b_{3} S_{1}\left\langle w_{1} b_{1} b_{4} T_{1}\right\rangle b_{5} \phi_{1}\right\rangle \\
& x_{3}=\left\langle b_{3} S_{1}\left\langle\mathrm{P}_{\mathrm{r}} T_{1} M_{6} w_{1}\right\rangle b_{5} \phi_{1}\right\rangle \\
& x_{4}=\left\langle 1\left\langle w_{1} \tau b_{1} S_{1}\right\rangle b_{5} \phi_{1}\right\rangle \\
& x_{5}=\left\langle T_{1}\left\langle w_{1} \tau b_{1} S_{1}\right\rangle b_{5} \phi_{1}\right\rangle \\
& x_{6}=\left\langle D \phi_{1}\left\langle w_{1} \quad b_{1} b_{4} T_{1}\right\rangle b_{4} D S_{1}\right\rangle, \\
& x_{7}=\left\langle D \phi_{1}\left\langle w_{1} D T_{1}\right\rangle b_{1} S_{1}\right\rangle, \\
& x_{8}=\left\langle D \phi_{1}\left\langle b_{1} T_{1} \quad M_{6} w_{1}\right\rangle b_{4} D S_{1}\right\rangle
\end{aligned}
$$

where $\langle u, v\rangle=\int_{-1 / 2}^{1 / 2} u v d z$ and $w_{1}, T_{1}, \phi_{1}$ and $S_{1}$ are trail functions that satisfy the boundary conditions. The above choice of trigonometry function tacitly implies the use of a higher order Galerkin method. For very large $M_{l}$, one gets the results for the magnetic mechanism, and the critical thermomagnetic Rayleigh number for stationary mode is calculated using

$$
N_{c}=M_{1} R_{c}=\frac{x_{1}-a^{2} R_{S}\left(x_{2} S_{T}+x_{3}\right)}{\left(1+M_{5}\right)\left[\left(1-S_{T}\right) \tau\left(x_{5}+x_{7}\right)+x_{6} S_{T}+x_{8}\right]} .
$$




\section{Discussion of results}

The linear stability analysis of Soret driven thermohaline convection in a ferromagnetic fluid layer heated from below and salted from above saturating a porous medium subjected to a transverse uniform magnetic field has been considered in the presence of temperature dependent viscosity by using the Brinkman model. Here the free-free boundary conditions are used. The present investigation is carried out through stationary instability. The small thermal perturbation technique is used and the normal mode technique is applied for the perturbation quantities.

Before we discuss the significant results of the system, we turn our attention to the possible range of values of various parameters arising in the study. The range of values of the temperature dependent viscosity parameter $V$ is assumed from 0.1 to 0.5 (Ramanathan and Muchikel, 2006). The ratio of magnitude to gravitational force $M_{l}$, is assumed to be 1000 (Finlayson, 1970). The range of salinity Rayleigh number $R_{S}$ is between -500 and 500 and Soret parameter $S_{\mathrm{T}}$ ranges from -0.002 to 0.002 (Sekar et al., 2013). The Brinkman model has been used for the permeability $k$ which ranges from 0.1 to 0.9 (Vaidyanathan et al., 2005) and the non-buoyancy magnetization parameter $M_{3}$ is taken from 5 to 25 (Sekar et al., 2013). For these type of fluids $M_{2}$ will have a negligible value and hence taken to be zero. The Prandtl number $\mathrm{P}_{\mathrm{r}}$ is taken to be 0.01 (Vaidyanathan et al., 2005) and the magnetic numbers $M_{4}, M_{5}$ and $M_{6}$ are assumed to be 0.1 (Sekar $e t$ al., 2013 and Sekar and Raju, 2013). The ratio of mass transport to heat transport $\tau$ is assumed from 0.03 to 0.011 (Sekar et al., 2006).

Figure 1 presents the plots of the critical magnetic thermal Rayleigh number $N_{\mathrm{C}}$ versus the nonbuoyancy magnetization parameter $M_{3}$ for different values of the temperature dependent viscosity $V, S_{\mathrm{T}}=$ - $0.002, R_{\mathrm{S}}=-500$ and $k=0.1$. It indicates that the non-buoyancy magnetization parameter $M_{3}$ has a destabilizing effect on the system when both $V$ and $M_{3}$ are increased. This is shown by a fall in $N_{C}$ values. This is because variation in magnetization releases extra energy which adds up to the thermal energy to destabilize the system.

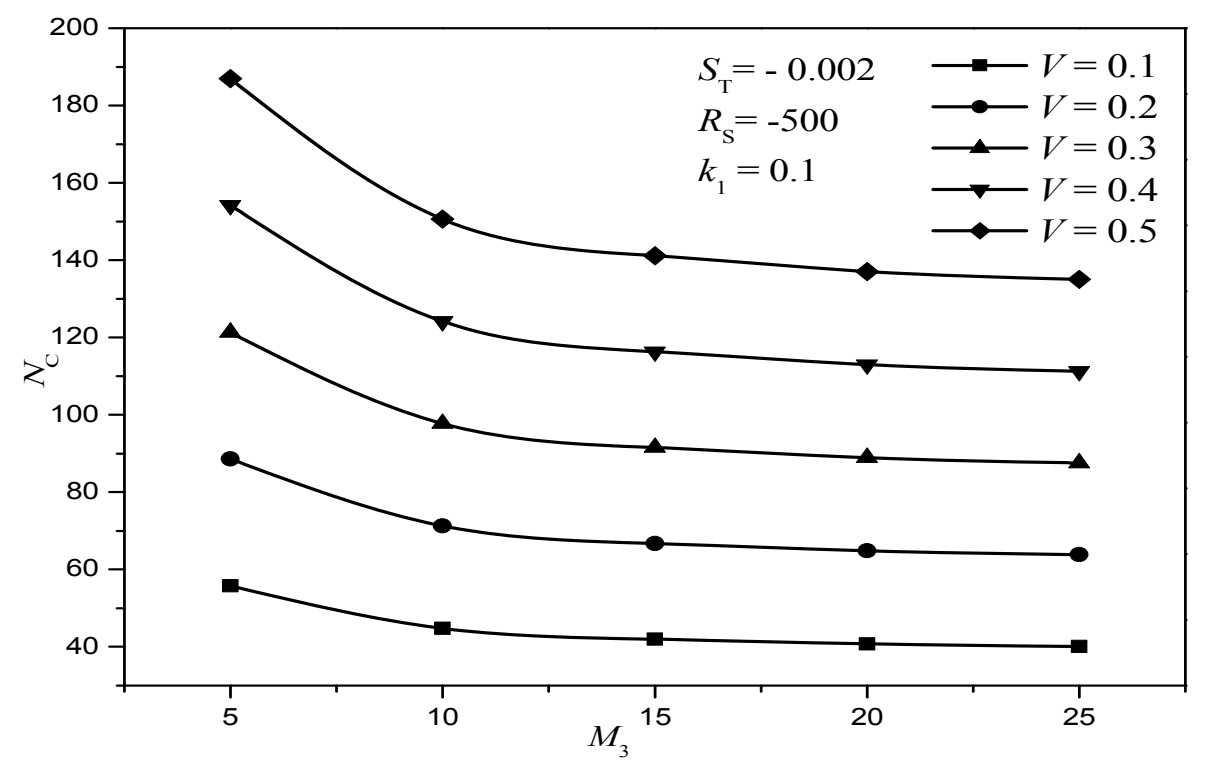

Fig.1. Marginal instability curve for variation of $N_{C}$ versus $M_{3}$ for different values of the temperature dependent viscosity $V$.

In Figs $2 \mathrm{a}$ and $\mathrm{b}$, the variation of the critical thermal magnetic Rayleigh number $N_{C}$ versus the temperature dependent viscosity $V$ for different permeability of the porous medium $k$. Both figures exhibit a destabilizing behavior because the presence of a porous medium increases from 0.1 to $0.9, N_{\mathrm{C}}$ decreases. It is also observed from the figures that the increase in the pore size makes the fluid flow easy to cause 
convection early. Figure 2a illustrates that as $k$ increases, $N_{C}$ decreases for a negative range of $R_{S}$ and $S_{T}$ and this behavior can also be observed exactly in the positive range of $R_{S}$ and $S_{T}$ in Fig.2b. Therefore, Figs 2a and $b$ illustrate the same destabilizing effect on the convective system.

(a)

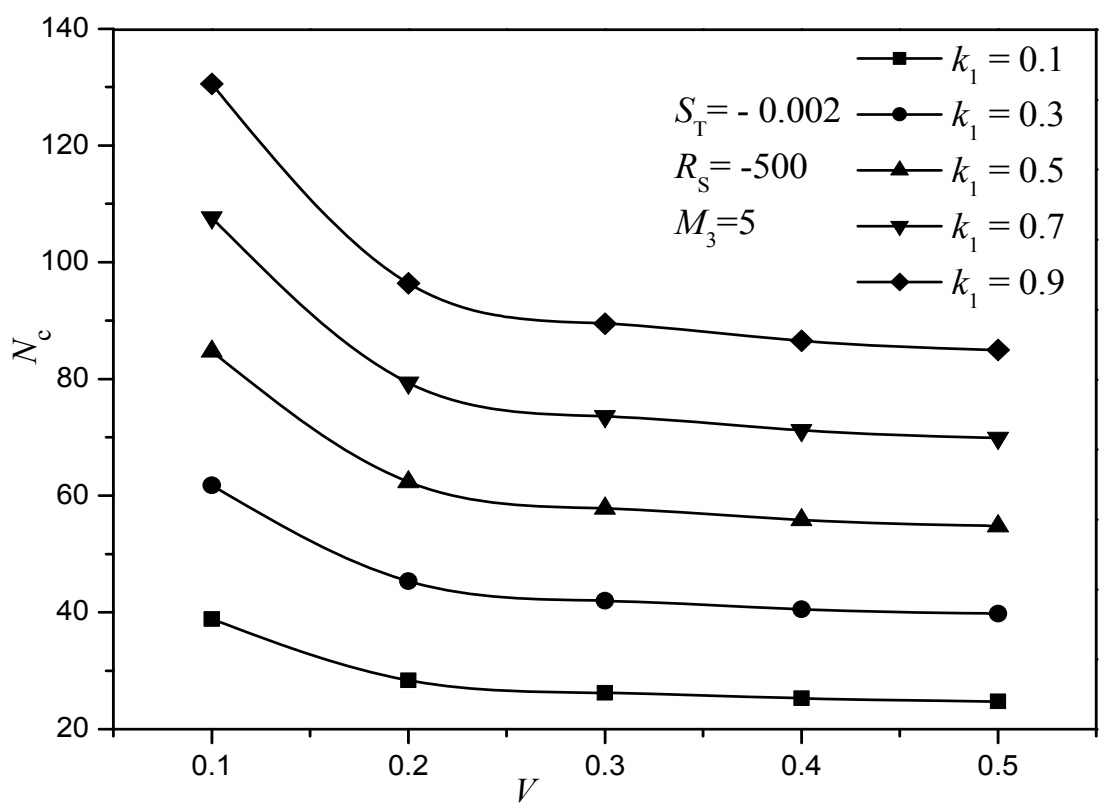

(b)

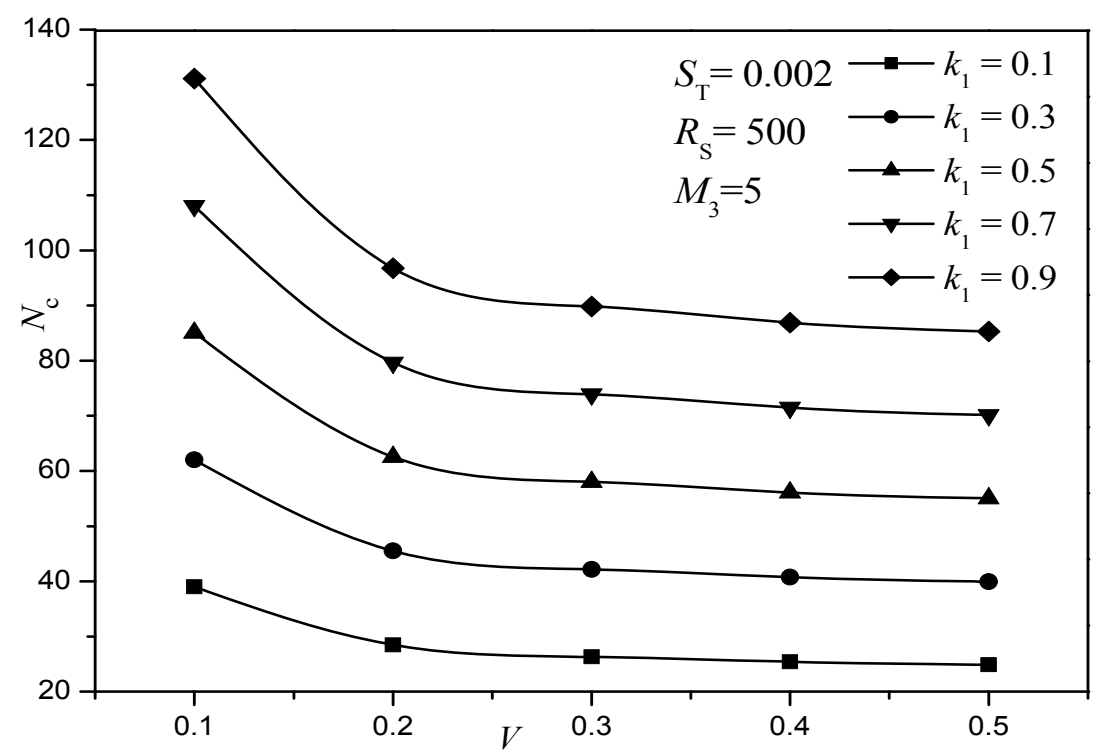

Fig.2. (a) Marginal instability curve for variation of $N_{C}$ versus $V$ for different values of the medium permeability $k, S_{T}=-0.002, R_{S}=-500, \tau=0.03 M_{3}=5$. (b) Marginal instability curve for variation of $N_{C}$ versus $V$ for different values of the medium permeability $k, S_{T}=0.002, R_{S}=500, \tau=0.03, M_{3}=5$.

Figures 3a, b and c represent $N_{\mathrm{C}}$ versus $V$ for different values of $R_{\mathrm{S}}, S_{\mathrm{T}}$ and $k$. It is observed from Figs $3 \mathrm{a}$ and $\mathrm{b}$ that the temperature dependent viscosity $V$ has a stabilizing effect on the system when $V$ increases, $N_{\mathrm{C}}$ increases and this stabilizing effect of $V$ is rather pronounced. Further, both Figs $3 \mathrm{a}$ and $\mathrm{b}$ are analyzed for different porous medium $k$. When the values of $k$ are 0.1 and 0.9 and negative value of $S_{T}$, the system show 
the same stabilizing behavior and there is no change in the convective system. Figure $3 \mathrm{c}$ illustrates $N_{C}$ versus $V$ for the positive value of $S_{T}$, due to the positive value of $S_{T}$ and $k=0.9$, the system has a non-equilibrium position compared with Figs $3 \mathrm{a}$ and b. Also in Fig.3c, the destabilizing behavior is not much pronounced when $V$ increases, $N_{\mathrm{C}}$ decreases.

Figures $4 \mathrm{a}, \mathrm{b}$ and $\mathrm{c}$ show the variation of $N_{\mathrm{C}}$ versus the interdiffusion of heat and mass, namely the Soret effect $S_{T}$ for different $V, R_{S}$ and $k$. Figures $4 \mathrm{a}$ and b give as increase of $S_{T}$, increase of $N_{C}$. This leads to stabilizing effect is not much pronounced. Figure $4 \mathrm{c}$ show that as $S_{T}$ increases, $N_{C}$ decreases. It is seen that the system destabilizes.

It is observed from Fig. 5 that the increase in the ratio of mass transport to the heat transport $\tau$ shows a stabilizing behavior, for an increasing value of $V$. When positive range of $R_{S}$ and $S_{T}$, the critical magnetic Rayleigh number $N_{C}$ has the equal value of the negative range of $R_{S}$ and $S_{T}$. This is because the increase in mass transport adds up to the system to be top heavy.

(a)

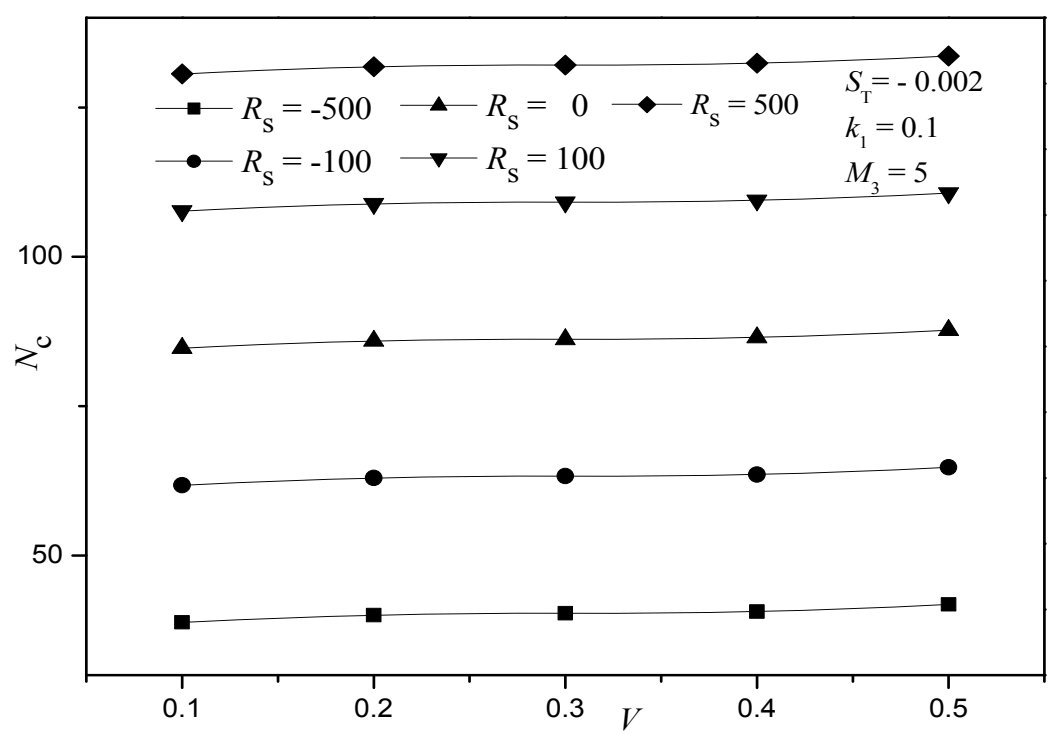

(b)

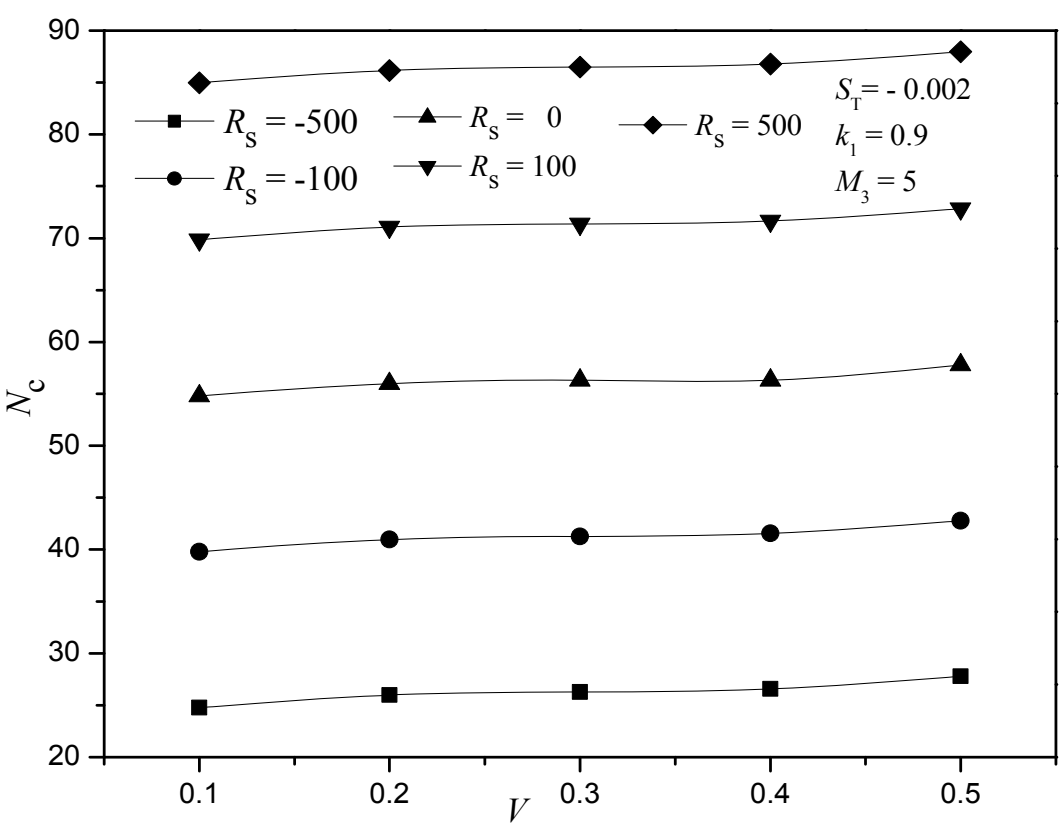


(c)

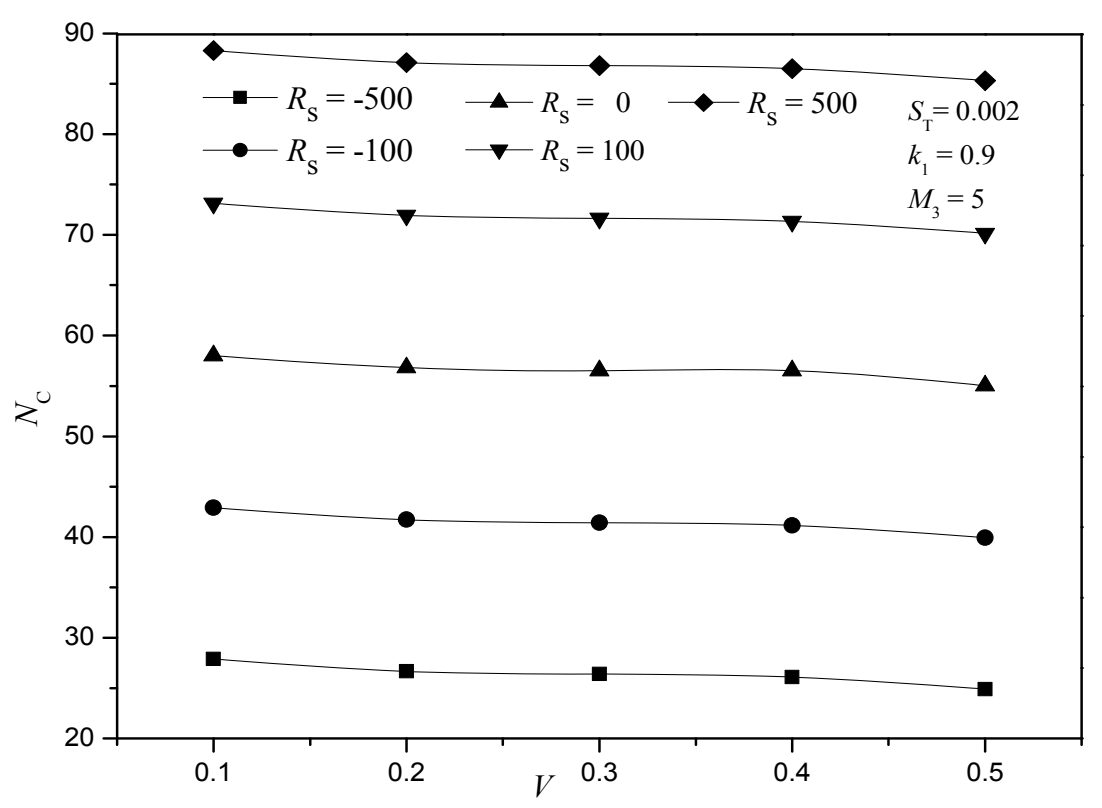

Fig.3. (a) Marginal instability curve for variation of $N_{\mathrm{C}}$ versus $V$ for different values of the salinity Rayleigh number $R_{S}, S_{T}=-0.002, M_{3}=5, \tau=0.03$ and $k=0.1$. (b) Marginal instability curve for variation of $N_{\mathrm{C}}$ versus $V$ for different values of the salinity Rayleigh number $R_{S}, S_{T}=-0.002, M_{3}=5, \tau=0.03$ and $k=$ 0.9. (c) Marginal instability curve for variation of $N_{C}$ versus $V$ for different values of the salinity Rayleigh number $R_{S}, S_{\mathrm{T}}=0.002, M_{3}=5, \tau=0.03$ and $k=0.9$.

(a)

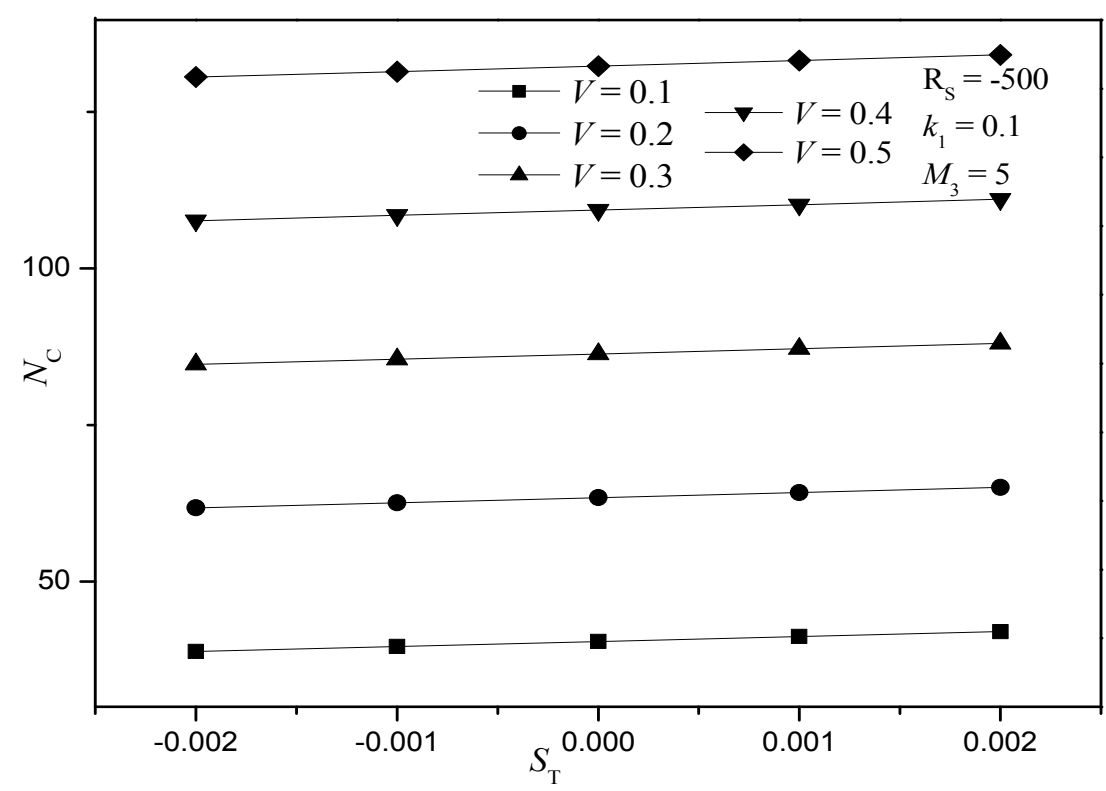


(b)

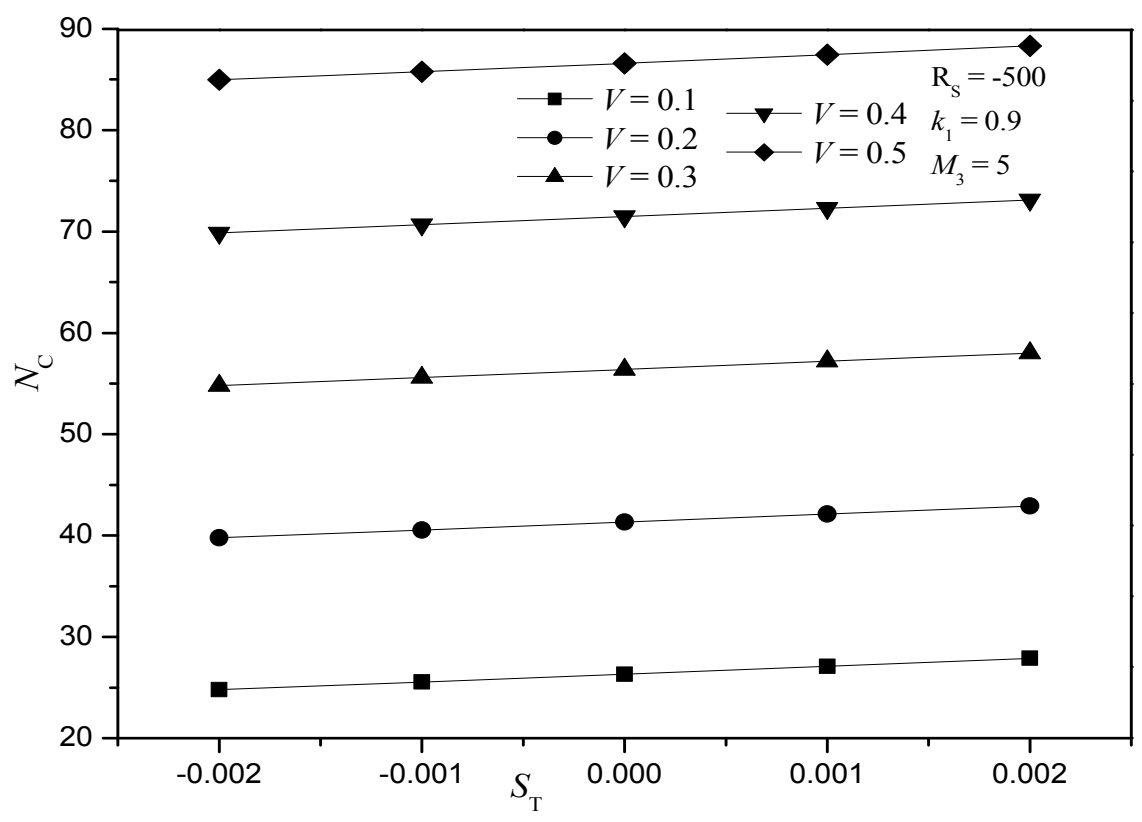

(c)

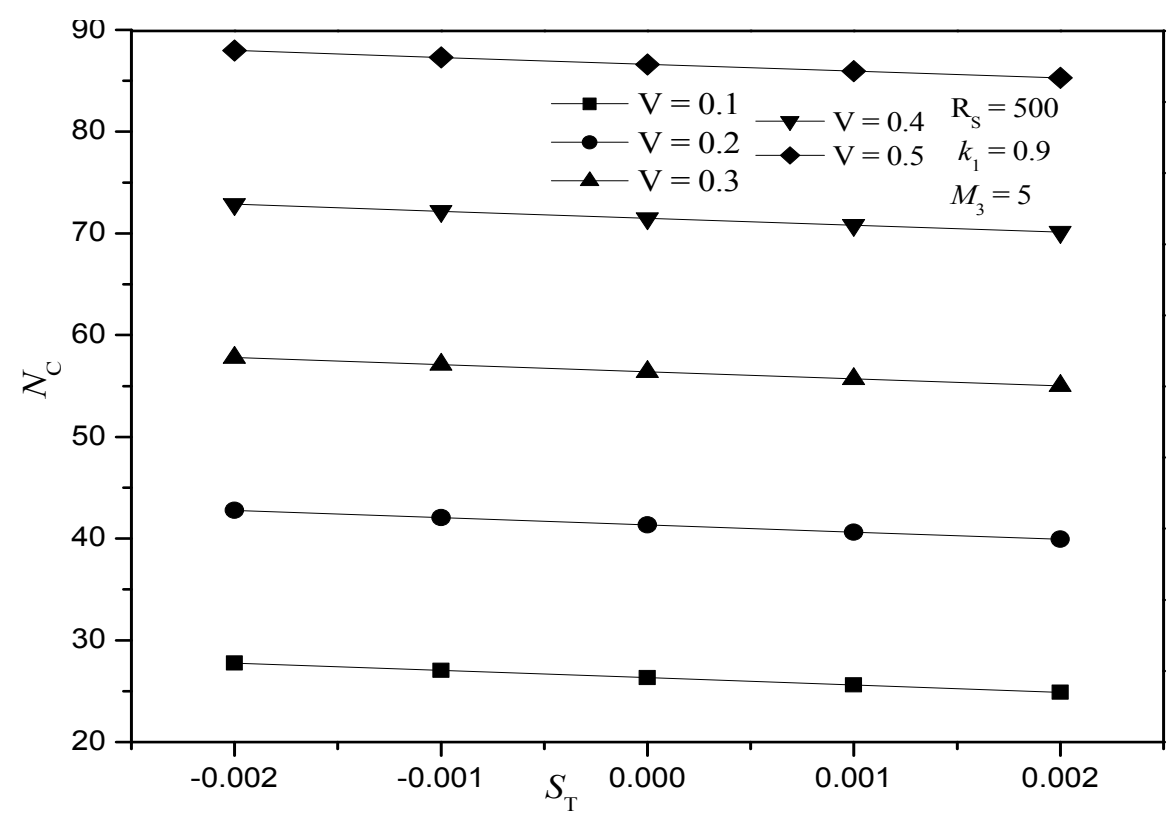

Fig.4. (a) Marginal instability curve for variation of $N_{\mathrm{C}}$ versus $S_{\mathrm{T}}$ for different values of the temperature dependent viscosity $V, R_{S}=-500, M_{3}=5, \tau=0.03$ and $k=0.1$. (b) Marginal instability curve for variation of $N_{\mathrm{C}}$ versus $S_{\mathrm{T}}$ for different values of the temperature dependent viscosity $V, R_{S}=-500, M_{3}=$ $5, \tau=0.03$ and $k=0.9$. (c) Marginal instability curve for variation of $N_{\mathrm{C}}$ versus $S_{\mathrm{T}}$ for different values of the temperature dependent viscosity $V, R_{S}=500, M_{3}=5, \tau=0.03$ and $k=0.9$. 


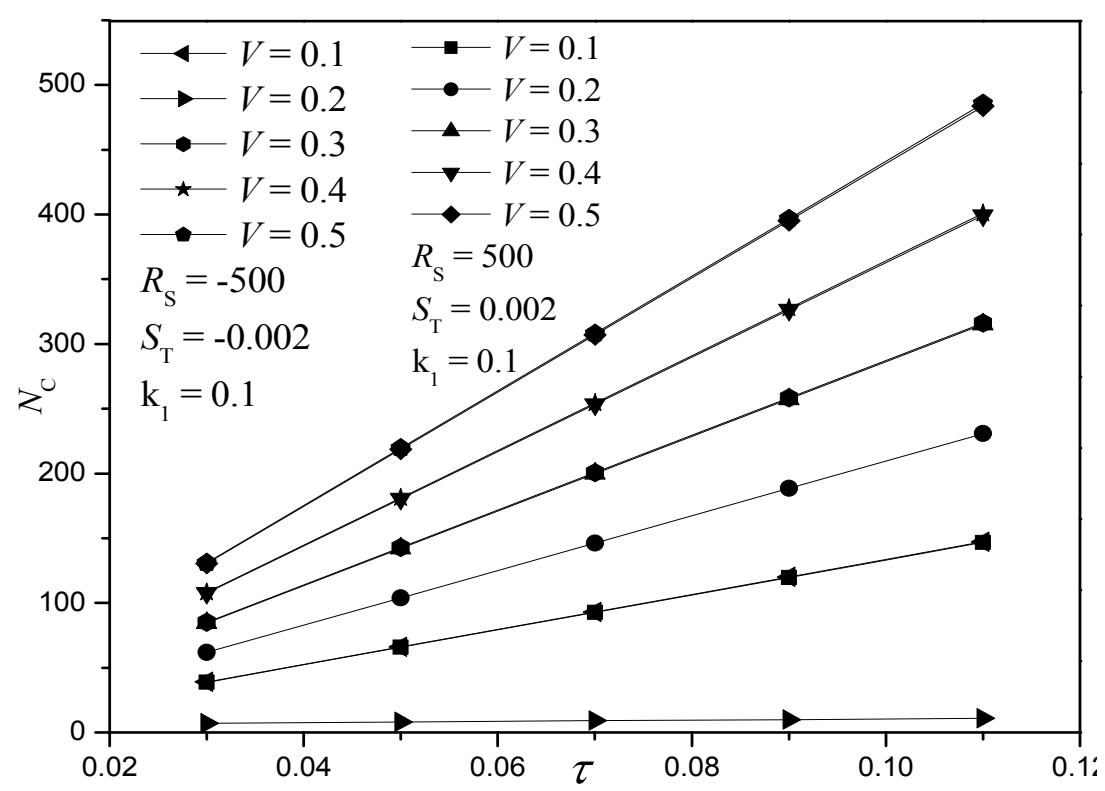

Fig.5. Marginal instability curve for variation of $N_{\mathrm{C}}$ versus $\tau$ for different values of the temperature dependent viscosity $V, M_{3}=5, k=0.1, R_{S}=-500, S_{T}=-0.002$ and $R_{S}=500, S_{T}=0.002$.

\section{Conclusion}

Soret-driven ferro thermoconvective instability of a magnetic fluid layer heated from below and salted from above in the presence of a porous medium and temperature field dependent viscosity suspended to a transverse uniform magnetic field has been investigated using the Brinkman model. The computational Galerkin method is applied. In this investigation, we have analyzed the effect of various parameters such as the medium permeability, buoyancy magnetization parameter, non- buoyancy magnetization parameter, ratio of mass transport to heat transport, temperature dependent viscosity parameter, Soret coefficient, salinity Rayleigh number and wave number.

The non-buoyancy magnetization parameter $M_{3}$ and the permeability of the porous medium $k$ have a destabilizing influence on the convective system. The stabilizing effect is investigated for the temperature dependent viscosity parameter $V$ in a very small value of salinity concentration and also the destabilizing behavior is analyzed for the temperature dependent viscosity parameter $V$ in a large value of salinity concentration.

Thus from the above analysis, one can conclude that the magnetization parameter, temperature gradient and salinity gradient have a profound influence on the onset of convection in a porous medium.

\section{Acknowledgement}

The authors are grateful to Prof. D. Govindarajulu, Principal, Pondicherry Engineering College, Puducherry for his constant encouragement. The author K. Raju is thankful to UGC for grant of Rajiv Gandhi National Fellowship 2010 - 2011 (Award letter number: F. 14-2(SC)/2010 (SA-III), Dated: May 2011).

\section{Nomenclature}

\footnotetext{
$\boldsymbol{B}$ - magnetic induction $T$

$C_{v, H}$ - effective heat capacity at constant volume and magnetic field $\left(\mathrm{kJ} / \mathrm{m}^{3} \mathrm{~K}\right)$
} 


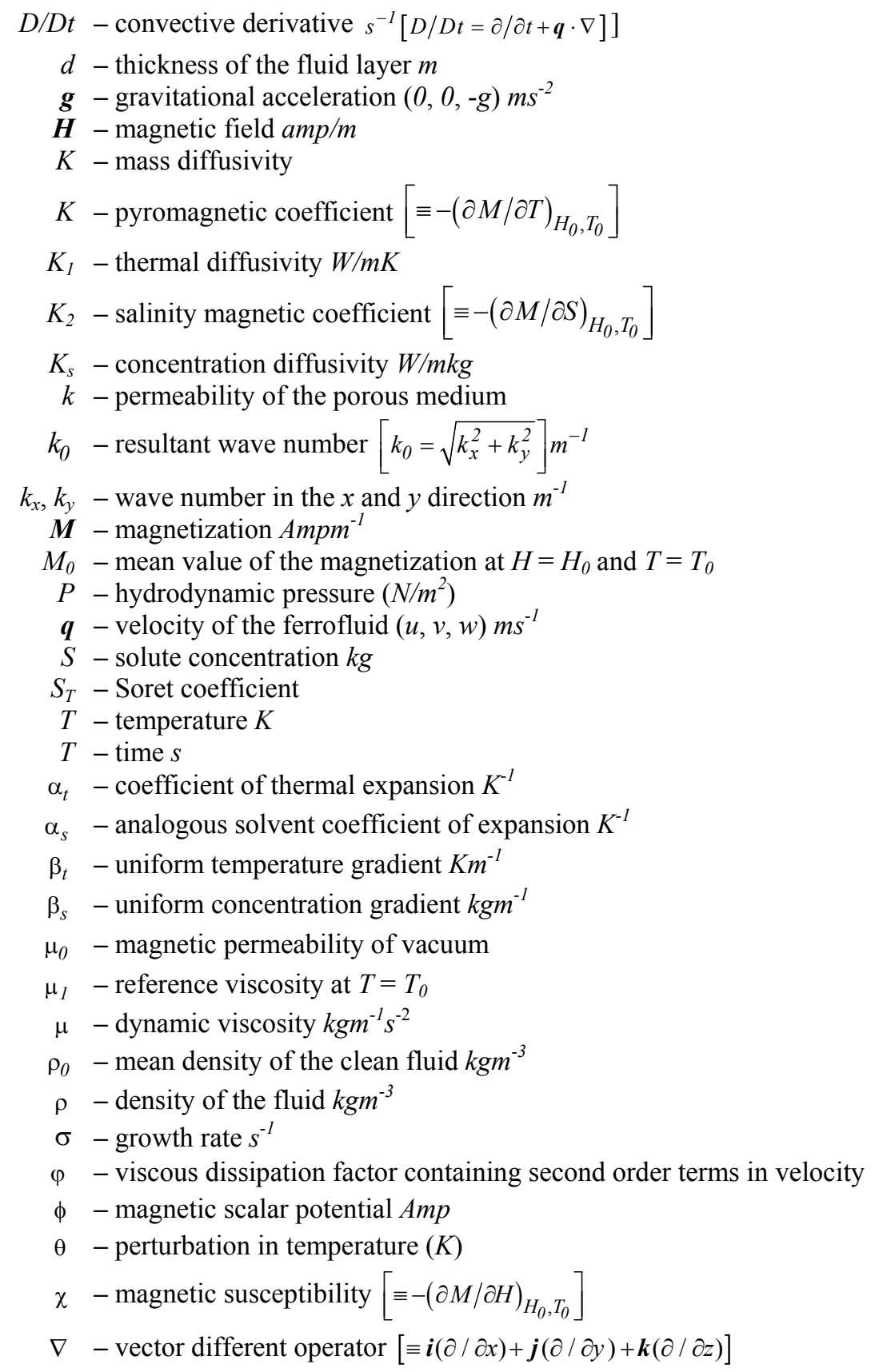

\section{References}

Baines P.G. and Gill S.E. (1969): On thermohaline convection with linear gradients. - Journal of Fluid Mechanics, vol.37, pp.289-306.

Berkovsky B. and Bastovoy V. (1996): Magnetic Fluids and Application Handbook. - New York: Begell House Publishers.

Chandrasekhar S. (1981): Hydrodynamics and Hydromagnetic Stability. - New York: Dover Publication.

Finlayson B.A. (1970): Convective instability of ferromagnetic fluids. - International Journal of Fluid Mechanics, vol.40, pp.753-767.

Gazeau F., Baravian C, Bacri J.C., Perzynski P. and Shiomis M.I. (1997): Physics Reviews - E 56, pp.614. 
Nanjundappa C.E., Shivakumara I.S. and Arunkumar R. (2010): Bénard-Marangoni ferroconvection magnetic field dependent viscosity. - Journal of Magnetism and Magnetic Materials, vol.322, pp.2256-2263.

Nanjundappa C.E., Shivakumara I.S. and Arunkumar R. (2012): Onset of Marangoni-Bénard ferroconvection with temperature dependent viscosity. - Microgravity Sci. Technol. DOI 10.1007/s 12217-012-9330-9.

Odenbach S. and Thurm S. (2012): Magnetoviscous Effects in Ferrofluids. - edited by Stefan Odenbach (SpringerVerlag, Berlin, Heidelberg).

Ramanathan A. and Muchikel N. (2006): Effect of temperature dependent viscosity on ferroconvection in a porous medium. - International Journal of Applied Mechanics and Engineering, vol.11, pp.93-104.

Rosensweig R.E. (1985): Ferrohydrodynamics. - Cambridge: Cambridge University Press.

Schwab L., Hildebrandt U. and Stierstadt K. (1983): Magnetic Bénard Convection. - Journal of Magnetism and Magnetic Materials, vol.39, pp.113-114.

Sekar R., Vaidyanathan G., Hemalatha R. and Senthilnathan S. (2006): Effect of sparse distribution pores in a Soretdriven ferro thermohaline convection. - Journal of Magnetism and Magnetic Materials, vol.302, pp.20-28.

Sekar R., Raju K. and Vasanthakumari R. (2013): A linear analytical study on Soret-driven ferrothermohaline convection in an anisotropic porous medium. - Journal of Magnetism and Magnetic Materials, vol.331, pp. $122-128$.

Sekar R., Raju K. and Vasanthakumari R. (2013a): Linear stability analysis of Coriolis force on ferrothermohalineconvection saturating an anisotropic porous medium with Soret effect. - Global Journal of Mathematical Analysis, vol.1(2), pp.37-47.

Sekar R. and Raju K. (2013): Effect of magnetic field dependent viscosity on Soret-driven thermoconvective instability of ferromagnetic fluid in the presence of rotating anisotropic porous medium of sparse particle suspension. International Journal of Mathematical Sciences, vol.12, pp.13-31.

Shivakumara I.S., Nanjundappa C.E. and Ravisha M. (2010): The onset of buoyancy-driven convection in a ferromagnetic fluid saturated porous medium. - Meccanica, vol.45, pp.213-223.

Siddheshwar P.G. (2004): Thermorheological effect on magneto convection in weak electrically conducting fluids and lg or $\mu g$. - Pramana J. Phys., vol.62, pp.61-68.

Stiles P.J. and Kagan M. (1990): Thermoconvective instability of a horizontal layer of ferrofluid in a strong vertical magnetic field. - Journal of Magnetism and Magnetic Materials, vol.85, pp.196-198.

Sunil, Chand P., Mahajan A. and Sharma P. (2011): Effect of rotation on double-diffusive convection in a magnetized ferrofluid with internal angular momentum. - Journal of Applied Fluid Mechanics, vol.4, pp.43-52.

Suresh Govindan., Vasanthakumari R. and Radja Sacravarthy P. (2012): Numerical study on the effect of temperature dependent viscosity on ferroconvection in an anisotropic porous medium. - International Journal of Engineering Technology and Advanced Engineering, vol.2, pp.51-55.

Vaidyanathan G., Sekar R. and Balasubramanian R. (1991): Ferroconvective instability of fluids saturating a porous medium. - International Journal of Engineering Sciences, vol.29, pp.1259-1267.

Vaidyanathan G., Sekar R. and Ramanathan A. (1995): Ferrothermohaline convection in a porous medium. - Journal of Magnetism and Magnetic Materials, vol.149, pp.137-142.

Vaidyanathan G., Sekar R. and Ramanathan A. (1997): Ferrothermohaline convection. - Journal of Magnetism and Magnetic Materials, vol.176, pp.321-330.

Vaidyanathan G., Sekar R., Hemalatha R., Vasanthakumari R. and Senthilnathan S. (2005): Soret-driven ferro thermohaline convection. - Journal of Magnetism and Magnetic Materials, vol.288, pp.460-469.

Received: August 13, 2013

Revised: December 15, 2013 